\title{
Tamoxifen: Pharmacokinetics and Pharmacodynamics
}

\section{Shahbaz $\mathbf{K}^{1,2,3 *}$}

${ }^{1}$ Center for Health Technology, Faculty of Science, University of Technology Sydney, Australia

${ }^{2}$ Department of pharmacy and pharmacology, University of Agriculture Faisalabad, Pakistan

${ }^{3}$ Director, Center for Breast Cancer, Department of Oncology, Perfect Health Pvt Ltd. Islamabad, Pakistan

*Corresponding Author: Kiran Shahbaz, Center for Health Technology, Faculty of Science, University of Technology Sydney, Australia; E-mail: dr.kiran.shahbazz@gmail.com

\section{Abstract}

Tamoxifen is the hormone therapy of choice in pre-menopausal estrogen receptor positive breast cancer in women and sometimes in post-menopausal women. The environmental and genetic factors widely affect the pharmacokinetic parameters of a drug and this effect is known as pharmgeonetics. The pharmacokinetic parameters that are what a body does with the drug, such as bioavailability, half-life, distribution and excretion, has primary importance in the treatment of a disease. The pharmacodynamic parameters which elaborate what a drug does with the body like side effects and positive symptoms are the pillars of therapeutic outcome. In depth knowledge of both parameters is essential to get maximum benefits from a treatment plan in a disease for example breast cancer. This review enlightens the pharmacological aspects of tamoxifen in detail.

Keywords: Pharmgeonetics; Tamoxifen; Pharmacokinetic; Pharmacodynamics

\section{Introduction}

\section{Breast Cancer}

Breast cancer is the second highly pervasive cause of mortalities in the world [1]. It does not cover only women but also men and food animals. Metastatic breast cancer is more drastic as the main causative agent is not known. Although, multiple causes have been known to be involved in the metastatic breast cancer, but still chemotherapy is a question to absolutely treat this cancer [1]. 1.3 million women suffer from breast cancer in United States. Similarly 1 out of every 7 women had breast cancer. Not only humans but also animals become victim of breast cancer.

\section{Tamoxifen}

Among the chemotherapeutic anti-estrogens, tamoxifen is the most essential as being used for 40 years worldwide. Yet there is no appropriate data for this medicine to support its entire kinetics and dynamics due to the environmental effects. Contrary to the previous decade when cervical cancer was the major cause of death now breast cancer accounts for highest morbidity and mortality rate in females [2]. The precise numbers of mortality and cancer cases in Pakistan are unknown [3]. Similar trend has been observed for Australia. If we follow standard treatment guidelines (STG) proposed by WHO, the therapeutic outcome of a disease may be altered significantly [4]. 
Tamoxifen was introduced by AstraZeneca of UK for the first time and being frequently prescribed as hormonal therapy for estrogen positive breast cancer in the clinics of Pakistan and Australia. It is far better to use right medicine with right dose at the right stage of the disease to opt maximum curative results. The exact kinetic and dynamic mechanism within human is necessary for manufacturing a drug against any disease (Figure 1).

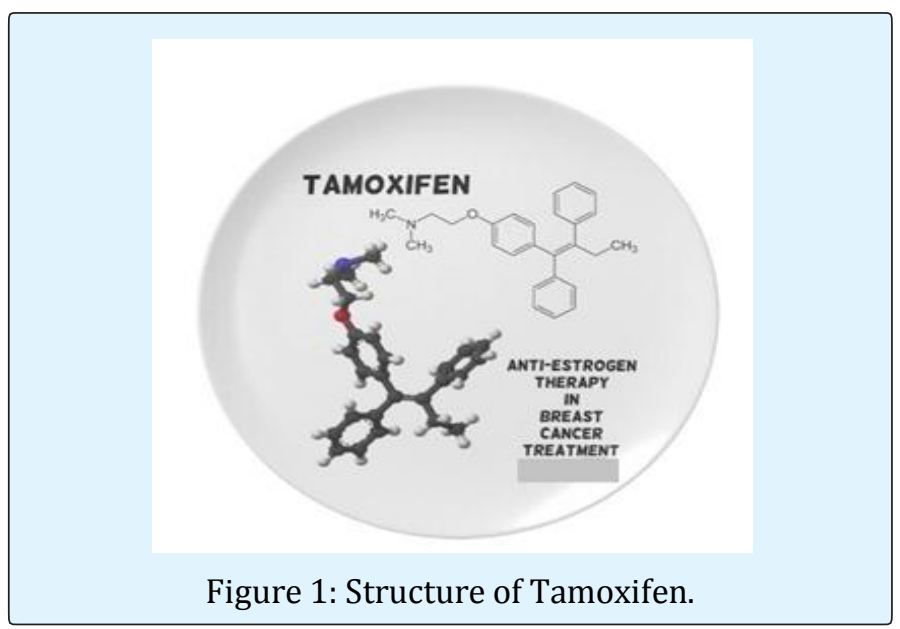

\section{Mode of Action}

Tamoxifen is being frequently prescribed as hormonal therapy of estrogen positive breast cancer in the clinics of Pakistan. There is a competitive binding of tamoxifen to estrogen receptors in cancer cells and other tissue targets, and this in turn produces a nuclear complex that decreases DNA synthesis and inhibits estrogen action. It is a non-steroidal agent with potential anti estrogenic abilities which occupies estrogen binding sites in breast tissue and metabolized by the liver enzyme Cyp2D6 (cytochrome P450, family 2, subfamily D, polypeptide 6), rendering active metabolites of Tamoxifen includes $\mathrm{N}$ desmethyl tamoxifen, endoxifen and 4 hydroxy tamoxifen (figure 2) [5].

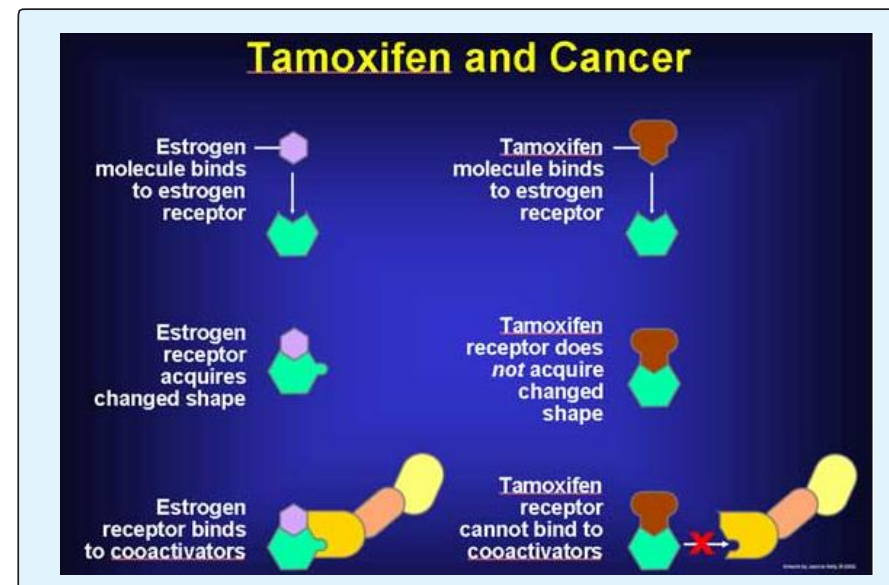

Figure 2: Mode of action of Tamoxifen.
Tamoxifen is known as a classic pro-drug requiring metabolic activation to start its pharmacological activity. Mainly 2D6 of cytochrome 450 appears to be the ratelimiting enzyme converting the pharmacologically inactive metabolites (Tamoxifen and NDM) to endoxifen, and further contributes to the formation of 4-OH-Tam from Tamoxifen. The findings depicts that both genetic and environmental (drug-induced) factors that change CYP2D6 enzyme activity affect Tamoxifen treatment results [5]. Research was done to check the breast density after use of Tamoxifen in patients. MRI of breast was performed after Tamoxifen use. It was concluded that with the use of Tamoxifen breast cancer is reduced hence the breast density came out to be less [6].

\section{Uses}

Long term use of Tamoxifen as prophylaxis is recommended and found useful with decrease in recurrence rate of breast cancer. The risk-lowering effect of Tamoxifen appears to persist for at least 10 years, yet most side effects of Tamoxifen do not continue after the 5year treatment period. Thus the long term treatment with Tamoxifen is appreciated [7].

Tamoxifen has been the adjuvant therapy of choice for postmenopausal, node-positive, and estrogen or progesterone receptor positive women since the mid1980s, and for postmenopausal, node-negative, and estrogen or progesterone receptor-positive women since the early 1990s. It is also being used in many cases of node-negative and receptor-positive premenopausal women. A high proportion (40-60\%) of all women who undergo potentially curative surgery for breast cancer now receive adjuvant Tamoxifen therapy for a period of 2 to 5 years. It was first approved for pharmaceutical use in the United Kingdom in 1973 and in the United States in 1977; Tamoxifen is presently registered in 97 countries. Tamoxifen use has been estimated at more than 7 million patient/years. The usual dose in the United States and the United Kingdom is $20 \mathrm{mg}$ /day for 1 to 2 years whereas in continental Europe, usual doses are 30 to $40 \mathrm{mg} /$ day [8].

Tamoxifen is used for the treatment of invasive breast cancer in men and women, the most common type of breast cancer, following surgery and/or radiation and for preventing invasive breast cancer in women at high risk for developing it. Tamoxifen also is used for the treatment of women following surgery and radiation for a less common type of breast cancer called ductal carcinoma in situ (DCIS or Intraductal Carcinoma). Women who have had DCIS are at high risk for developing invasive breast cancer at a later date, and tamoxifen prevents development of the invasive cancer in almost half of the 


\section{Open Access Journal of Pharmaceutical Research}

women during the first five years of treatment. Occasionally, tamoxifen is used to stimulate ovulation.

Widespread use of tamoxifen has invited efforts to develop routine assays for this drug and its metabolites in human plasma. Procedures based on gas chromatography with mass spectrometry are highly specific and involve equipment not generally available. In 1994, Fried \& Wainer [9] reported a HPLC assay that incorporates post column fluorescent activation and that avoids problems related to the variable photochemical degradation of the phenanthrenes. The method reported by Lee, et al. represents an evolution of the Fried \& Wainer method with three major differences: the incorporation of an internal standard in the assay, the use of a liquid-liquid extraction procedure to further purify and allow concentration of the sample, and the quantification of 4hydroxy-N-desmethyl Tamoxifen, a metabolite that may have anti-cancer activity. Unfortunately, the total run time achieved was about $60 \mathrm{~min}$. The pharmaceutical preparations of Tamoxifen citrate available in Brazil are based on different excipients and some coating films, which may not reflect the real bioavailability described for these tablets.

Human volunteers are usually used in bioavailability tests for determination of the plasmatic concentrations of the studied medication. In this study, healthy dogs were used as animal model in substitution to humans, due to the low cost and the satisfactory results in comparison to human volunteers. It was also verified that the traditional methods for determination of the plasmatic concentration of the tamoxifen are generally costly, in which several advanced equipments are used. Thus, a consistent and cost effective analytical methodology was adapted from literature. Experiment performed on dogs for the pharmacokinetic of Tamoxifen showed that the literature of drug on dogs does not fits on human beings due to GI changes and other physiological facts. Thus these studies suggest need for Tamoxifen pharmacokinetics in human beings separately to get the pharmacokinetic parameters.

I6\% of the study participants were 65 years of age or older and 6\% were at least 70 years of age. Reduction in the incidence of breast cancer was observed in women receiving Tamoxifen across all age groups. In this trial, the risk of serious adverse effects (e.g., endometrial cancer, deep-vein thrombosis, pulmonary embolism and stroke) was at highest in women 50 years of age and older [10].

\section{Side Effects}

Like other chronic diseases, the treatment of breast cancer can be at a better stance if there is more patient compliance and less side effects. Most common side effects caused by tamoxifen are nausea, hot flashes, vaginal dryness, loss of sexual desire. It is notable that tamoxifen is not an antagonist at all tissues like in breast tissue. It therefore has agonist effect in bones and ovaries. In addition it does not have a cardioprotective effect on heart and causes thromboembolism and fatty liver. It shows a reduction in libido and evidences of decreased cognition have been reported. Figure 3 shows the side effect of tamoxifen at mitochondrial level which states an increased reactive oxygen species pathway due to tamoxifen within mitochondria of a cell leading to apoptosis [11]. Whilst it may also prove that the tamoxifen may leads to aging process as it is being used by breast cancer patients for more than 5 years.

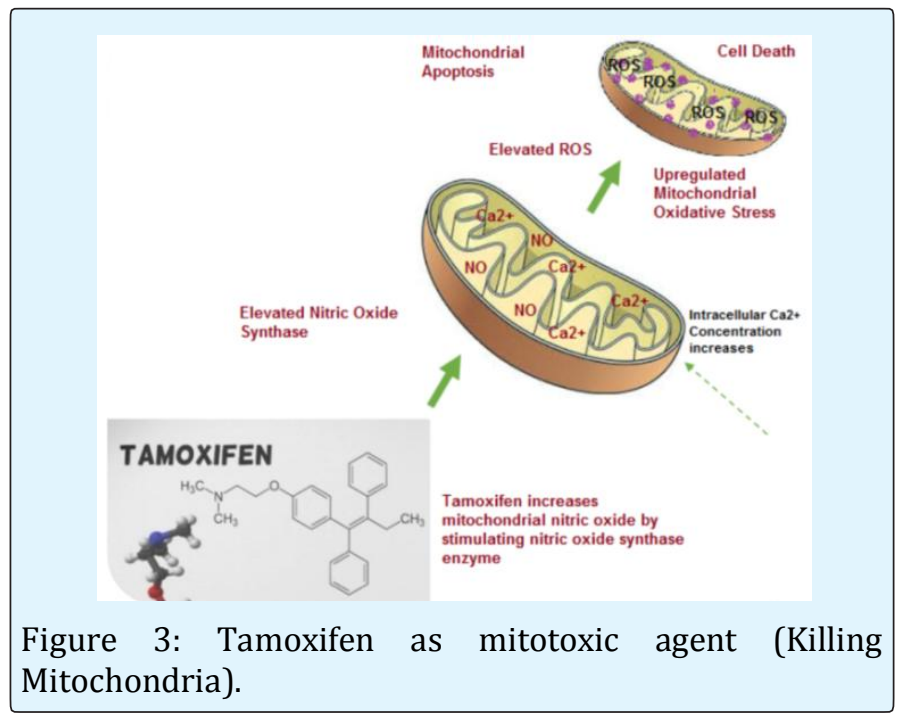

\section{Less Common or Rare Side Effects}

Less common side effects include anxiety, blistering, peeling, or loosening of the skin and mucous membranes, blurred vision, cataracts in the eyes or other eye problems, change in vaginal discharge, chest pain, chills, confusion, cough, dizziness, fainting, fast heartbeat, fever, hoarseness, lightheadedness, lower back or side pain, pain or feeling of pressure in the pelvis, pain or swelling in the legs, pain, redness, or swelling in your arm or leg, painful or difficult urination, rapid shallow breathing, shortness of breath or trouble with breathing, skin rash or itching over the entire body, sweating, weakness or sleepiness, wheezing, vaginal bleeding and yellow eyes or skin. Some of the not known incidences include bloating, constipation, darkened urine, diarrhea, difficulty with breathing, indigestion, itching, joint or muscle pain, large hard skin blister, large hive-like swelling on the face, eyelids, lips, tongue, throat, hands, legs, feet, and sex organs, loss of appetite, nausea, pain in the stomach or side, possibly radiating to the back, red irritated eyes, red skin lesions, often with a purple center, sore throat, sores, 


\section{Open Access Journal of Pharmaceutical Research}

ulcers or white spots in the mouth or on the lips, unusual tiredness or weakness and vomiting.

Some side effects of tamoxifen may occur that usually do not require medical attention. These side effects may go away during treatment as your body adjusts to the medicine. Also, your health care professional may be able to tell you about ways to prevent or reduce some of these side effects. Check with your health care professional if any of the following side effects continue or are bothersome or if you have any questions about them:

\section{More Common Side Effects}

More common side effects are absent, missed, or irregular periods and decrease in the amount of urine. There seems to be correlation between long-term Tamoxifen administration and endometrial proliferation [12]. Mild-moderate gastrointestinal toxicity (diarrhea, anorexia) and reversible neurotoxicity were observed in dogs receiving chemotherapy plus high-dose Tamoxifen given for seven days [13]. Nausea and vomiting can occur. Dizziness, lethargy, depression, irritability and cerebellar dysfunction have been described.

The lowest cumulative dose of Tamoxifen known to have induced retinopathy, an adverse effect which is recognized to be dose-dependent, is $7.7 \mathrm{~g}$ [14].

\section{Dosage Regimen}

In some animal species, oestrogenic agonist effects become effective at dosages equivalent to 10-100 times the human therapeutic dose [15]. Relevant in vitro data is not available yet.

The dose for metastatic breast cancer treatment, DCIS, and prevention of breast cancer is $10 \mathrm{mg}$ twice daily or 20 mg once daily for 5 years. The dose for stimulation of ovulation is 5-40 mg twice daily for 4 days. Tamoxifen can be taken with food. An overview of the adjuvant tamoxifen clinical trials revealed that the efficacy of 20 $\mathrm{mg} /$ day of tamoxifen was equivalent to that of higher doses of the drug (that is, 30 to $40 \mathrm{mg}$ /day). The Hormone Replacement Therapy Opposed by Low Dose Tamoxifen (HOT) study is a multicenter phase III trial in postmenopausal healthy women, current or de novo HRT users, randomized to either tamoxifen $5 \mathrm{mg}$ /day or placebo for 5 years, with 5 years of follow-up. The trial is designed to assess whether the combination of HRT and low-dose tamoxifen retains the benefits while reducing the risks of either agent. The HOT trial completed its accrual in 2007, with 1,884 subjects enrolled. An Italian multicenter phase III trial is currently ongoing to evaluate the efficacy and safety of tamoxifen $5 \mathrm{mg} /$ day for reducing breast cancer incidence in 1,400 women operated [16]. As a prophylactic agent for high risk patients, the approved dose of Tamoxifen is $20 \mathrm{mg}$ daily given for five years. $20 \mathrm{mg}$ Tamoxifen on day 2 to day 5 of the menstrual cycle is recommended. For patients with irregular menstrual cycle, Tamoxifen is given on any day. If menstruation occurs, dosage is then given every day 2 of the cycle. If there is no response, a subsequent dose is given 45 days after the initial treatment.

A study revealed that the dosage regimen of tamoxifen should be managed as to attain steady state levels within the plasma to achieve therapeutic results. Furthermore, it was advised to decrease the dosage interval after administering $20 \mathrm{mg}$ tablet of tamoxifen in healthy female volunteers of Pakistan [17]. However, pharmgeonetic effect is notable here, thus, similar study should be revised in other global regions for maximum therapeutic outcomes. Decensi and colleagues performed two experiments to assess the effect of low-dose tamoxifen in 127 healthy hysterectomized women aged 35 to 70 years, randomly assigned to placebo $(n=31)$ or tamoxifen 20 $\mathrm{mg} /$ day $(n=30)$ (first experiment), and tamoxifen 10 $\mathrm{mg} /$ day $(n=34)$ or tamoxifen $10 \mathrm{mg}$ alternate days $(n=$ 32) (second experiment) [18].

\section{Bioavailability}

Fuchs et al., did work on the pharmacokinetics of Tamoxifen plus bioavailability study depicts that further experiments should be performed on Tamoxifen due to its longer half -life. As the sampling time increases, pharmacokinetic parameters become more accurate and vice versa.

\section{Metabolism}

According to a work on urine samples of Tamoxifen detected through HPLC it was observed that the metabolites are contained in the urine but not in all samples. This thing clearly depicts that the metabolism of Tamoxifen varies in various individuals.

In Tamoxifen treated patients, there was a weak evidence that the poor-metabolizer variant, CYP2D6*6 $(\mathrm{MAF}=0.01)$, was associated with decreased BCSS $(\mathrm{P}=$ $0.02 ; \mathrm{HR}=1.95 ; 95 \% \mathrm{CI}=1.12-3.40)$. No other variants, including CYP2D6*4 (MAF $=0.20)$, previously reported to be associated with poorer clinical outcomes, were associated with differences in BCSS, in either the Tamoxifen or non-Tamoxifen groups. CYP2D6*6 may affect BCSS in Tamoxifen-treated patients. However, the 


\section{Open Access Journal of Pharmaceutical Research}

absence of an association with survival in more frequent variants, including CYP2D6*4, questions the validity of the reported association between CYP2D6 genotype and treatment response in breast cancer. Until larger, prospective studies confirming any associations are available, routine CYP2D6 genetic testing should not be used in the clinical setting [19].

The excretory fate of Tamoxifen and its metabolites has not been well characterized. Following oral administration of $20 \mathrm{mg}$ dose of radio labeled Tamoxifen in women, normally $65 \%$ of the administered dose was excreted in feces for a 2-week time period, mainly as polar conjugates; unchanged tamoxifen and unconjugated metabolites accounted for less than $30 \%$ of the fecal radioactivity. Unchanged tamoxifen and $\mathrm{N}$-desmethyl tamoxifen have been detected in urine in small amounts. In animals, Tamoxifen and/or its metabolites appear to undergo extensive enterohepatic circulation and are excreted in feces and urine as glucuronides, other conjugates, and unidentified polar metabolites [20].

The densitometric analytic procedure used for Tamoxifen can also be used to quantify its desmethyl metabolite. In a study involving six healthy male volunteers, Tamoxifen tablets were shown to be as bioavailable as a solution of Tamoxifen citrate. After administration of a single dose of $20 \mathrm{mg}$, peak serum levels of Tamoxifen were $42 \mathrm{ng} / \mathrm{ml}$; those of the metabolite were $12 \mathrm{ng} / \mathrm{ml}$. The half-lives of the drug and metabolite were approximately 4 and 9 days, respectively, after a single dose. After three widely separated single doses, a reversible increase in elimination half-life occurred [21].

In breast cancer patients discontinuing chronic Tamoxifen therapy, the serum elimination of metabolites $\mathrm{X}, \mathrm{Y}$ and $\mathrm{E}$ paralleled that of Tamoxifen, whereas that of metabolite $\mathrm{Z}$ did not. The serum elimination of Tamoxifen and metabolites $\mathrm{X}$ and $\mathrm{B}$ was increased by aminoglutethimide treatment, whereas that of metabolites $\mathrm{Z}, \mathrm{Y}$, and $E$ was not [22]. The half-life is $7-14$ hours. In $30.4 \mathrm{mg}$ of Tamoxifen citrate tablet, $20 \mathrm{mg}$ of active ingredient is found. After a single oral tablet of $20 \mathrm{mg}$ of Tamoxifen shows an average peak plasma concentration of $40 \mathrm{ng} / \mathrm{mL}$ (range 35 to $45 \mathrm{ng} / \mathrm{mL}$ ) observed approximately five hours after dosing. The decline in plasma concentrations of Tamoxifen is biphasic with a terminal elimination halflife of about 5 to 7 hours [23].

Tamoxifen is extensively metabolised after oral administration. $\mathrm{N}$-desmethyl Tamoxifen, the major metabolite found in patients' plasma, undergoes secondary metabolism to 4-hydroxy-N-desmethyl
Tamoxifen (endoxifen). The enzyme involved in this conversion is cytochrome P450 2D6 (CYP2D6), which also converts Tamoxifen to 4-hydroxy Tamoxifen. This metabolite undergoes secondary metabolism to endoxifen. It is widely accepted that the majority of the anti-proliferative effect of Tamoxifen occurs via its active metabolites. 4-hydroxy Tamoxifen has at least 100 -fold greater affinity for the ER than Tamoxifen, and has a similarly increased potency in anti-proliferative action. Endoxifen has an equivalent anti-proliferative potency and ER binding ability to 4-hydroxy Tamoxifen but is present in higher concentrations in the plasma. Any factor that diminishes production of these metabolites could impact on Tamoxifen efficacy. Several enzymes are involved in these metabolic pathways, with CYP2D6 playing a pivotal role. CYP2D6 is a polymorphic gene with over 90 documented alleles [24].

Another research on the urine sample of Tamoxifen taking patients demonstrated that the patient profile varies significantly for Tamoxifen levels. Thus this may be due to some hepatic enzymes or other kidney functions. However it was clear that patient had different levels of Tamoxifen in their urine samples [25].

\section{Absorption}

Peak concentrations occur 4-7 h after oral dosing. Peak concentrations after single oral doses of $20 \mathrm{mg}$ are about $40 \mu \mathrm{g} / \mathrm{l}$. There is no information on absolute bioavailability [26-28].

\section{Biological Half-Life}

The elimination is biphasic, with an initial half-life of around $7 \mathrm{~h}$ and a terminal half-life of 7-11 days [24-26].

\section{Distribution}

Tamoxifen is more than $99 \%$ protein-bound in serum, predominantly to albumin. In patients with breast cancer, concentrations of Tamoxifen and its metabolites in pleural, pericardial and peritoneal effusion fluid are between 20 and $100 \%$ of those in serum, but only trace amounts enter the cerebrospinal fluid. Concentrations in breast cancer tissue exceed those in serum. The volume of distribution is $50-60 \mathrm{l} / \mathrm{kg}[27,28]$.

\section{Genetic Variations}

Genetic variations in Cyp-2D6 enzyme among various people have shown $20 \%$ of decrease in the pharmacological action of tamoxifen [29]. It is a prodrug and its active metabolites may only support an 


\section{Open Access Journal of Pharmaceutical Research}

antiestrogen therapy as they change by Cyp-3A4 and Cyp2D6 [30]. Genotype therefore has an essential implication on the outcomes of patients taking tamoxifen as breast cancer therapy. The effect of enzyme activity on the pharmacology of a drug due to variations in the environmental factors is known as pharmenzymonetic [4].

\section{Toxicity}

There is no information on the acute toxicity of tamoxifen in over dosage. The lowest cumulative dose of tamoxifen known to have induced retinopathy, an adverse effect which is recognized to be dose-dependent, is $7.7 \mathrm{~g}$ [14]. There is no data available for children toxicity. If we on the data of animals for the toxicity of tamoxifen, in some animal species, oestrogenic agonist effects become manifest at dosages equivalent to 10-100 times the human therapeutic dose [15]. Similarly there is no in vitro relevant data available for the toxicity experiments of tamoxifen.

\section{Carcinogenicity}

A case-control study showed a significantly increased relative risk of carcinoma of the uterus in women previously treated with tamoxifen AND who had previously had radiotherapy involving the uterus. The study showed an increase in relative risk with tamoxifen treatment alone which was NOT statistically significant [31].

\section{Teratogenicity}

Studies in neonatal male and female mice at relative doses 10 times higher than those used in humans have shown genital tract abnormalities similar to those caused by diethylstilboestrol, a known transplacental carcinogen (diethylstilboestrol causes vaginal adenosis, which predisposes to clear cell carcinoma) [32-33].

\section{Mutagenicity}

Tamoxifen is believed not to be mutagenic [26].

\section{Interactions}

Tamoxifen agonize the anticoagulant effect of warfarin, and this interaction can be life-threatening [34]. This is because the coagulation of blood in the major arteries of major organs such as brain, heart and lungs may cause sudden death.

\section{Conclusion}

Tamoxifen is widely used anti breast cancer medicine prescribed in Australia and Pakistan. The parameters of tamoxifen has been well experimented in the world including pharmacokinetic and pharmacodynamic parameters, whilst a further study about its pharmgeonetic factor is required. Which is how various environmental factors affect the bioavailability and other pharmacological factors of tamoxifen. It is predicted that dosage regimen according to pharmgeonetic literature will increase the therapeutic results and wellbeing of a patient after using tamoxifen in breast cancer treatment [35].

Funding: The Australian President's Award (APA) equivalent The UTS President's scholarship (UTSP) and The UTS International Research Scholarship (UTS IRS)

Provider: University of Technology Sydney, 15 Broadway, Ultimo NSW 2007, Australia.

\section{References}

1. Shahbaz K, Mehfooz A, Khadam W, Din MU, Shahbaz $\mathrm{K}$, et al. (2014) Breast Cancer Vaccination- An Envisioned Future. IAJPR 4(3): 1580-1585.

2. Jemal A, Bray F, Center MM, Ferlay J, Ward E, et al. (2011) Global Cancer Statistics. Ca Cancer J Clin 61(2): 69-90.

3. Hanif M, Zaidi P, Kamal S, Hameed A (2009) Institution-based Cancer Incidence in a Local Population in Pakistan: Nine Year Data Analysis. Asian Pacific Journal of Cancer Prevention 10(2): 227230.

4. Shahbaz K (2015) Comparison between Standard Treatment Guidelines of Preeclampsia Proposed By Who And Current Practice in Tertiary Care Centers. WJPPS 4(8): 1566-1593.

5. Fuchs WS, Leary WP, van der MMJ, Gay S, Witschital K, et al. (1996) Pharmacokinetics and Bioavailability of Tamoxifen in Postmenopausal healthy women. Arzneimittelforschung 46(4): 418-422.

6. Chen JH, Yeun CC, Daniel C, Yi TW, Ke N, et al. (2011) Reduction of breast density using tamoxifen treatment evaluated by 3D MRI. Magon reson imaging 29(1): 91-98.

7. Cuzick J, Forbes JF, Sestak I, Cawthorn S, Hamed H, et al. (2007) Long-Term Results of Tamoxifen Prophylaxis for Breast Cancer 96-Month Follow-up of 


\section{Open Access Journal of Pharmaceutical Research}

the Randomized IBIS-I Trial. J Natl Cancer Inst 99(4): 272-282.

8. Guelen P, Stevenson D, Briggs R, De Vos D (1987) The bioavailability of Tamoplex (tamoxifen). Part 2. A single dose cross-over study in healthy male volunteers. Methods Find Exp Clin Pharmacol 9(10): 685-90.

9. Fried KM, Wainer IW (1994) Direct determination of tamoxifen and its four major metabolites in plasma using coupled column high-performance liquid chromatography. J Chromatogr B Biomed Appl 655(2): 261-268.

10. Zafra-Ceres M, De Haro T, Farez-Vidal E, Blancas I, Bandres F, et al. (2013) Influence of CYP2D6 polymorphisms on serum levels of tamoxifen metabolites in Spanish women with breast cancer. Int J Med Sci 10(7): 932-937.

11. Nazarewicz RR, Zenebe WJ, Parihar A, Larson SK, Alidema E, et al. (2007) Tamoxifen Induces Oxidative Stress and Mitochondrial Apoptosis via Stimulating Mitochondrial Nitric Oxide Synthase. Cancer Res 67(3): 1282-1290.

12. Uziely B, Lewin A, Brufman G, Dorembus D, Mor-Yosef $S$ (1993) The effect of tamoxifen on the endometrium. Breast Cancer Res Treat 26(1): 101-105.

13. Waddle J, Fine R, Case B, Trogdon M, Tyczkowska K, et al. (1999) Phase I and pharmacokinetic analysis of high-dose tamoxifen and chemotherapy in normal and tumor-bearing dogs. Cancer Chemother Pharmacol 44(1): 74-80.

14. Griffiths M (1987) Tamoxifen retinopathy at low dosage. Am J Ophthalmol 104(2): 185-186.

15. ABPI (1989) Cesamet. ABPI Data Sheet Compendium 1989-90 pp. 819-820. London: Datapharm Publications Ltd.

16. Decensi A, Robertson C, Viale G, Pigatto F, Johansson $\mathrm{H}$, et al. (2003) A randomized trial of low-dose tamoxifen on breast cancer proliferation and blood estrogenic biomarkers. J Natl Cancer Inst 95(11): 779-790.

17. Shahbaz K, Muhammad F, Aslam B, Shahbaz K, Javed I (2015) Disposition Kinetics and Dosage Regimen of Tamoxifen in Adult Healthy Female Volunteers. WJPPS 5(3): 67-81.
18. Decensi A, Bonanni B, Guerrieri-Gonzaga A, Gandini S, Robertson C, et al. (1998) Biologic activity of tamoxifen at low doses in healthy women. Journal of the National Cancer Institute 90(19): 1461-1467.

19. Abraham J, Maranian, Driver KE, Platte R, Kalmyrzaev B, et al. (2010) CYP2D6 gene variants: association with breast cancer specific survival in a cohort of breast cancer patients from the United Kingdom treated with adjuvant Tamoxifen. Breast Cancer Res 12(4): R64.

20. De Santana DP, Rossana MCB, Ruth S, Miracy MA, César GB, et al. (2008) Reversed phase HPLC determination of Tamoxifen in dog plasma and its pharmacokinetic after a single oral dose administration. Quim Nova 31(1): 47-52.

21. Adam HK, Patterson JS, Kemp JV (1980) Studies in the metabolism and pharmacokinetics of Tamoxifen in normal volunteers. Cancer Treat Rep 64(6-7): 761764.

22. De Vos D, Slee PH, Stevenson D, Briggs RJ (1992) Serum elimination half-life of tamoxifen and its metabolites in patients with advanced breast cancer. Cancer Chemother Pharmacol 31(1): 76-78.

23. Furr BJ, Jordan VC (1984) The pharmacology and clinical uses of tamoxifen. Pharmacol Ther 25(2): 127-205.

24. Golander Y, Sternson LA (1980) Paired-ion chromatographic analysis of Tamoxifen and two major metabolites in plasma. J Chromatogr 181(1): 41-49.

25. Carter SJ, Li XF, Mackey JR, Modi S, Hanson J et al. (2001) Biomonitoring of Urinary Tamoxifen and its metabolites from breast cancer patients using nonaqueous capillary electrophoresis with electrospray mass spectrometry. Electrophoresis 22(13): 27302706.

26. Martindale (1999) The Complete Drug Reference (33rd edn), Pharmaceutical Press, London, pp: 2315.

27. Buckley MMT, Goa KL (1989) Tamoxifen. Drugs 37(4): 451-490.

28. Lien EA, E Solheim, OA Lea, S Lundgren, S Kvinnsland, PM Ueland (1989) Distribution of 4-hydroxy-NdesmethylTamoxifen and other Tamoxifen metabolites in human biological fluids during Tamoxifen treatment. Cancer Res 49(8): 2175-2183. 
29. Schroth W, Goetz MP, Hamann U, Fasching PA, Schmidt M, et al. (2009) Association between CYP2D6 polymorphisms and outcomes among women with early stage breast cancer treated with tamoxifen. JAMA 302(13): 1429-1436.

30. Desta Z, Ward BA, Soukhova NV, Flockhart DA (2004) Comprehensive evaluation of tamoxifen sequential biotransformation by the human cytochrome P450 system in vitro: prominent roles for CYP3A and CYP2D6. J Pharmacol Exp Ther 310(3): 1062-1075.

31. Hardell L (1988) Tamoxifen as risk factor for carcinoma of corpus uteri. The Lancet 332(8610): 563.
32. Taguchi O (1987) Reproductive tract lesions in male mice treated neonatally with tamoxifen. Biol Reprod 37(1): 113-116.

33. Taguchi O, Nishizuka Y (1985) Reproductive tract abnormalities in female mice treated neonatally with tamoxifen. Am J obstet Gynecol 151(5): 675-678.

34. Ritchie LD, Grant S (1989) Tamoxifen-warfarin interaction: the Aberdeen hospitals drug file. BMJ 298(6682): 1253 .

35. Shahbaz K (2016) Pharmenzymonetics and Pharmgeonetics: A New Door in Pharmacology. WJPPS 5(2): 1424-1432. 